\title{
Comment on "Breaking wave induced cross-shore tracer dispersion in the surf zone: Model results and scaling",
}

\author{
Stephen M. Henderson ${ }^{1,2}$
}

Received 8 June 2007; accepted 23 July 2007; published 8 November 2007.

Citation: Henderson, S. (2007), Comment on "Breaking wave induced cross-shore tracer dispersion in the surf zone: Model results and scaling,' J. Geophys. Res., 112, C11005, doi:10.1029/2007JC004378.

[1] The mixing of tracer patches by nearshore breaking waves was investigated by Feddersen [2007, hereinafter referred to as F07]. Key results of F07 for periodic breaking waves, derived from numerical experiments, include: (1) the mean cross-shore tracer location changes little with mixing, (2) the variance of the cross-shore tracer position increases linearly with time, (3) the skewness of the cross-shore tracer position tends to zero as mixing progresses. Here, these results are derived analytically.

[2] F07 models tracer mixing using the diffusion equation (his equation (4))

$$
\partial_{\hat{t}} \hat{\phi}=\partial_{\hat{x}}\left[\hat{\kappa} \partial_{\hat{x}} \hat{\phi}\right]
$$

where $\hat{x}=$ (cross-shore distance) $/ L_{\kappa}, L_{\kappa}$ is the half-width of the breaking front-face of a wave (a "bore"), $\hat{t}=$ time $\times$ $\kappa_{0} L_{\kappa}{ }^{-2}, \kappa_{0}$ is the maximum diffusivity associated with breaking, and $\hat{\kappa}=$ diffusivity $/ \kappa_{0}$. Bore-generated mixing is modeled by narrow, shoreward propagating regions of high eddy diffusivity. Each bore propagates past a fixed location in a dimensionless time of order $\Delta \hat{T}=\hat{c}^{-1}$ (corresponding to dimensional time $\Delta T=L_{\kappa} / c$, where $c$ is the dimensional phase speed, and $\left.\hat{c}=c L_{\kappa} / \kappa_{0}\right)$. From (1), the variation in $\hat{\phi}$ during the passage of a single bore is

$$
\phi^{\prime} \sim \frac{\hat{\phi} \Delta \hat{T}}{L_{\hat{x}}^{2}}=\frac{\hat{\phi}}{\hat{c} L_{\hat{x}}^{2}}
$$

where $L_{\hat{x}}$ is the dimensionless patch width (i.e., the ratio between patch width and bore width). For large $\hat{c} L_{\hat{x}}^{2}, \phi_{\hat{\phi}}^{\prime}$ becomes small, and the instantaneous tracer concentration $\hat{\phi}$ roughly equals its wave-averaged value

$$
\bar{\phi}=\frac{1}{\hat{T}} \int_{\hat{t}-\hat{T} / 2}^{\hat{t}+\hat{T} / 2} \hat{\phi} d \hat{t}^{\prime}
$$

where $\hat{T}$ is the dimensionless wave period.

\footnotetext{
${ }^{1}$ Cooperative Institute for Limnology and Ecosystem Research, University of Michigan, Ann Arbor, Michigan, USA.

${ }^{2}$ Now at School of Earth and Environmental Sciences, Washington State University, Vancouver, Washington, USA.
}

Copyright 2007 by the American Geophysical Union. 0148-0227/07/2007JC004378
[3] Averaging both sides of (1) yields

$$
\partial_{\hat{t}} \bar{\phi}=\partial_{\hat{x}}\left[\bar{\kappa} \partial_{\hat{x}} \bar{\phi}+\overline{\kappa^{\prime} \partial_{\hat{x}} \phi^{\prime}}\right]
$$

where $\hat{\kappa}$ has been divided into wave-averaged and fluctuating components. From (2) and (4)

$$
\partial_{\hat{t}} \hat{\phi}=\partial_{\hat{x}}\left[\bar{\kappa} \partial_{\hat{x}} \hat{\phi}\right]+O\left(\hat{c}^{-1} L_{\hat{x}}^{-2}\right) .
$$

Given the scaling of F07, section $2.4, \hat{c}$ is $O(1)$, so the error in (5) is $O\left(L_{\hat{x}}^{-2}\right)$. Consequently, the dispersion of tracer patches whose width is much greater than the width of a bore is governed by a diffusion equation, with an effective diffusivity equal to the time-averaged diffusivity. For spatially uniform mean diffusivity $\bar{\kappa}$, solutions to (5) for the cross-shore tracer distribution approach a Gaussian with zero mean velocity, zero skewness, and tracer location variance increasing linearly with time, consistent with the results of F07. From (5) and the idealized bore diffusivity introduced in F07, section 2.2, for large $\hat{T}$ (i.e., for bore width $\ll$ wavelength)

$$
\bar{\kappa}=\frac{1}{\hat{T}} \int_{\hat{t}-\hat{T} / 2}^{\hat{t}+\hat{T} / 2} \hat{\kappa} d \hat{t}^{\prime} \approx \frac{1}{\hat{T}} \int_{-\infty}^{\infty} \exp \left[-\frac{\left(\hat{c} \hat{t}^{\prime}\right)^{2}}{2}\right] d \hat{t}^{\prime}=\frac{\sqrt{\pi}}{\hat{c} \hat{T}} .
$$

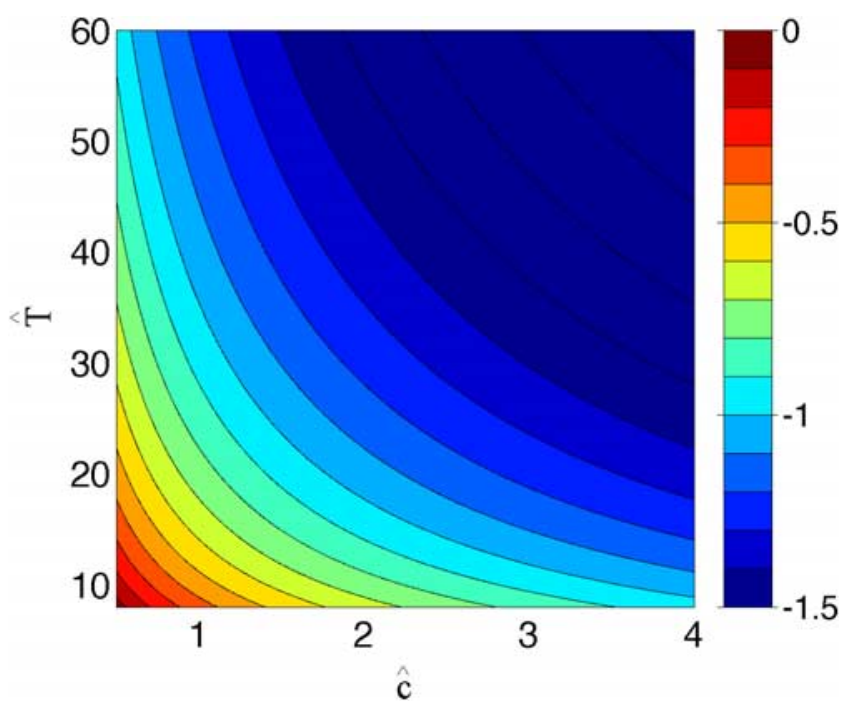

Figure 1. Contour plot of $\log _{10}(\alpha)$ predicted by (6) as a function of $\hat{c}$ and $\hat{T}$. Interval between contours is 0.1 . 
Predictions of (6) are consistent with the numerical results of F07 (compare Figure 1 of this comment with Figure 8 of F07, note $\alpha=2 \kappa$ ). In less idealized models (such as the Boussinesq model of F07, section 4$) \bar{\kappa}$ is not exactly given by (3) but remains $O\left(\hat{c}^{-1} \hat{T}^{-1}\right)$. Given the scaling of F07, section 2.4 , the dimensional mean diffusivity is of order $h^{2}$, $T$, where $h$ is the water depth and $T$ is the wave period.

[4] Acknowledgments. Funding was provided by the NOAA Great Lakes Environmental Research Laboratory (contribution 1433), the University of Michigan O.V.P.R., the Office of Naval Research, and the National Science Foundation. I thank Falk Feddersen for valuable discussions.

\section{References}

Feddersen F. (2007), Breaking wave induced cross-shore tracer dispersion in the surfzone: Model results and scalings, J. Geophys. Res., 112, C09012, doi:10.1029/2006JC004006.

S. M. Henderson, School of Earth and Environmental Sciences, Washington State University, 14204 NE Salmon Creek Avenue, Vancouver, WA 98686, USA. (shenders@coast.ucsd.edu) 